\title{
Calendar of Events
}

Association of Children's Prosthetic-Orthotic Clinics

2012 Annual Meeting

April 11-14, 2012

Banff, Alberta, Canada

Website http://www.acpoc.org/

American Academy of Neurology

64th Annual Meeting

April 21-28, 2012

New Orleans, LA

Website http://www.aan.com/go/press/ampress

American Occupational Therapy Association

2012 Annual Conference \& Expo

April 26-29, 2012

Indianapolis, IN

Website http://www.aota.org/ConfandEvents/ 2012Conference.aspx

The Pediatric Orthopedic Society of North America

2012 POSNA Annual Meeting

May 16-19, 2012

Denver, Colorado

Website

http://www.posna.org/meetings/anmeet/anmeet.asp

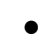

European Academy of Childhood Disability

24th Annual Meeting of the European Academy of Childhood Disability

\section{May 17-19, 2012}

Istanbul, Turkey

Website

http://www.eacd.org/meetings-detail.php?id=41

American Urological Association

AUA Annual Meeting

May 19-24, 2012

Atlanta, GA

Website http://www.aua2012.org/

Society for Research into Hydrocephalus and Spina Bifida

56th Annual Scientific Meeting

\section{May 2012}

Amsterdam, Netherlands

Website http://srhsb.org/meetings-and-events/ forthcoming-meetings-.aspx

American Physical Therapy Association

2012 Annual Conference \& Exposition

\section{June 6-9, 2012}

Tampa, FL

Website http://www.apta.org/AnnualConference/ 
The American Academy of Cerebral Palsy and Developmental Medicine (AACPDM) 65th Annual Meeting

\section{September 12-15, 2012}

Westin Harbour Castle Toronto, Ontario, Canada

Website: http://www.aacpdm.org/meetings/

American Society of Hand Therapists (ASHT)

ASHT's 35th Annual Meeting

October 18-21, 2012

$$
\text { San Diego, CA }
$$

Website http://www.asht.org/meeting/

62nd Congress of Neurological Surgeons Annual Meeting

\section{October 6-10, 2012}

Chicago, IL

Website: http://www.neurosurgeon.org/
Closing the Gap

30th Annual Conference

October 17-19, 2012

Minneapolis, MN

Website: http://www.closingthegap.com/conference/

American Academy of Pediatrics

AAP National Conference and Exhibition (NCE)

October 20-23, 2012

Ernest N. Morial Convention Center

New Orleans, LA

Website: http://www.aapexperience.org/

American Academy of Physical Medicine and Rehabilitation (AAPM \& R)

Annual Assembly and Technical Exhibition

November 15-18, 2012

Georgia World Congress Center

Atlanta, GA

Website: aapmr.org/assembly/pages/aapmr-2012annual-assembly.aspx 